\title{
EL USO DE FUENTES DOCUMENTALES PARA LA ENSEÑANZA DE LA HISTORIA LOCAL DE CÓRDOBA. EL CATASTRO DE ENSENADA COMO RECURSO DIDÁCTICO
}

\section{THE USE OF DOCUMENTARY SOURCES FOR THE TEACHING OF THE LOCAL HISTORY OF CORDOBA, SPAIN. THE CATASTRO DE ENSENADA AS A DIDACTIC RESOURCE}

\author{
Rafael Guerrero Elecalde \\ rgelecalde@uco.es \\ Departamento de Didácticas Específicas \\ Universidad de Córdoba (España)
}

Recibido: 06/04/2020

Aceptado: $14 / 09 / 2020$

\begin{abstract}
Resumen:
El objetivo del artículo reside en la publicación de los resultados de una actividad innovadora desarrollada en el Grado en Educación Primaria de la Universidad de Córdoba sobre el uso de fuentes documentales históricas, a través de plataformas digitales, para la enseñanza de la historia local. De esta manera, nos proponemos saber si este tipo de prácticas son útiles y mejoran los procesos de enseñanza-aprendizaje, en este caso, en la formación de los futuros docentes de Educación Primaria. La documentación seleccionada fue el Catastro de Ensenada y sus respuestas generales (Córdoba, 1752). Posteriormente a la actividad, se preguntó al alumnado, a través de un formulario de Google Forms, para reflexionar sobre los contenidos y el diseño de la docencia de las Ciencias Sociales.
\end{abstract}

Palabras clave: fuentes documentales; didáctica de las ciencias sociales; innovación pedagógica; métodos de enseñanza

\begin{abstract}
:
The main objective of this article is publishing the results of an innovative activity that was carried out with students of the Primary Education Degree from the University of Cordoba, using the documentary sources through online site for teaching local history. This means that we expect to know whether this kind of practices are useful and improve the teaching and learning process, and if so, improve their own of the future graduates of Primary Education. The documentation selected was the Cadastre of Ensenada and its general responses (Córdoba, 1752). Subsequently, students were asked, using Google Forms, to deliberate about the content and the teaching design of Social Sciences.
\end{abstract}

Keywords: documentary sources; Didactics of Social Sciences; pedagogical innovation; teaching methods 


\section{Introducción}

En este texto se recogen los resultados sobre una experiencia de innovación docente desarrollada con alumnado en formación del Grado de Educación Primaria de la Universidad de Córdoba durante el curso 2018/2019. Su objeto principal fue el diseño y ejecución de una práctica de innovación educativa que permita mejorar la calidad de la enseñanza de contenidos de Ciencias Sociales, por medio de la utilización de fuentes documentales y el estudio de la historia local. Para esta innovación se decidió trabajar con las respuestas generales de la ciudad de Córdoba del Catastro de Ensenada (1752).

Posteriormente, una vez desarrollada la implementación de la actividad innovadora en el aula, se quiso conocer, a través de un cuestionario, la opinión del alumnado sobre la experiencia didáctica realizada. El análisis de las respuestas mostró la eficacia de este tipo de prácticas en la mejora de los procesos de aprendizaje de la historia porque, por un lado, el trabajo con los documentos (fuentes desde donde se escribe la historia) permite el desarrollo del pensamiento histórico: una construcción compleja de los procesos del pasado, con sus vinculaciones con el presente. Una reflexión sin duda que es favorecida por el estudio de la historia local, que ayuda al alumnado a descubrir las evidencias pretéritas de su entorno más próximo, más concreto, abriéndole la puerta a concebir grandes dinámicas históricas, las que están más relacionadas con conceptos mucho más abstractos.

Igualmente, además de la conciencia histórica, con esta innovación se han desarrollado destrezas correspondientes con la competencia digital, la competencia de aprender a aprender y la competencia matemática.

\section{Marco teórico}

La historia local es un excelente vehículo para enseñar los procesos del pasado. Desde 1950, en el curriculum de los países anglosajones se han estado incluyendo su estudio como pieza principal en los procesos de enseñanza de las Ciencias Sociales (Vázquez de Prada, 1987; Rogers, 1972). Sin embargo, a pesar de estas iniciativas, estos presupuestos no fueron secundados en el continente porque prevaleció el concepto de que su aprendizaje no permitía ni el arraigo ni la motivación entre el estudiantado de Educación Primaria. Así ocurrió en Francia o en Alemania, por ejemplo (Prats, 1996).

En España, excepto en algunos momentos durante la Segunda República, también se continuó con estos planteamientos que obviaron la historia local hasta que, en la década de 1970, los estudios del entorno se convirtieron en la base principal de los contenidos del curriculum. Por entonces, los colectivos de renovación educativa, vinculados a EGB, respaldaron que los conocimientos históricos eran parte de los contenidos sociales, por lo que trabajaron por la incorporación de la enseñanza del medio en Educación Primaria (Vega, 1997). Como campo teórico, abrazaron el planteamiento de J. Piaget (1977), que defiende que exclusivamente se puede trabajar en esas edades contenidos determinados.

Algunos de ellos incluso acuñaron el término de "historia próxima", de forma parecida a como Schools Council History 13-16 Project utilizó el de "la historia a nuestro alrededor". En ambos casos, se ponía el acento en la proximidad o cercanía a la experiencia directa del alumno, tanto del tema a trabajar como de los materiales o fuentes para su realización. Esta concepción abarcaría la historia local propiamente dicha; la historia local reciente; la historia oral local; la historia familiar e incluso la historia del mismo alumno; y las manifestaciones locales de la historia general o estatal (Domínguez, 1986). 
En estos conceptos, el aprendizaje de la historia local vincula de forma directa lo concreto, conocido e inmediato con nociones más complicadas y abstractas. Por eso, se trata de una técnica apropiada para trabajar cuestiones correspondientes con la investigación como, por ejemplo, el establecimiento de hipótesis, la comprobación y concreción del saber.

El éxito inicial de estas propuestas se prolongó tras el establecimiento de la LOGSE, que potenció los estudios de historia local y del medio, convirtiéndose de este modo en uno de los ejes principales del área de Ciencias Sociales de Educación Primaria (Moral, Pérez, Torremocha y Valls, 1999).

Sin embargo, las nuevas tendencias pedagógicas, las cuales han sido refrendadas por la publicación de nuevas leyes educativas, han provocado el abandono de su enseñanza. En este momento ha quedado apartada tras otros contenidos disciplinares de la Didáctica de las Ciencias Sociales, muchos de ellos muy similares a los modelos tradicionales (Prats, 1996).

Por todo ello, parece inevitable reflexionar sobre los beneficios de la enseñanza y aprendizaje de la historia local para replantear su instalación en las aulas y el uso de recursos, más novedosos y atractivos para el alumnado, que favorezcan su impulso.

Pero, ¿qué beneficios tiene la enseñanza de la historia local? Principalmente, es una herramienta muy útil para establecer un diálogo entre mundos diferentes que se encuentran interconectados: lo particular y lo general, lo concreto y lo abstracto y fenómenos locales con fenómenos comunes (Clío 92, 1995; Aktekin, 2010; Pedreño, 2015). Una observación completa desde el punto de vista local ayuda a una aproximación a la materialidad espacial de las grandes construcciones sociales, así como a la comprensión de sus propios procesos.

También, su estudio favorece la averiguación de los principios de las estructuras y su transcurso cotidiano, aparte de conferir noticias precisas sobre los modelos generales (Pedreño, 2015). Por eso, favorece colocar los resultados del aprendizaje en un contexto más amplio, ya que el alumnado comprenderá que los conocimientos, las habilidades y las técnicas que están adquiriendo a través del estudio del medio los llevará a comprender dinámicas amplias del pasado. Igualmente, permite que los estudiantes adquieran competencias para introducirse en la interpretación histórica, siempre y cuando se les ayude con la incorporación de los conceptos teóricos apropiados. Asimismo, favorece la curiosidad del medio y a su vez el interés por el conocimiento de la historia (Domínguez, 1986).

Desde el punto de vista pedagógico, la historia de la localidad y, en general, los estudios del entorno pueden constituir un punto de interdisciplinariedad y motor de aprendizajes instrumentales base para la comunicabilidad. Además, cuando el estudiante se siente comprometido con la construcción y apropiación de su historia, empieza a tener las herramientas necesarias para construir identidades y un superior entendimiento del pasado (Gómez y García, 2018).

Por último, y continuando con las palabras del profesor J. Prats, la historia local permite a los estudiantes descubrir las evidencias históricas de su espacio más próximo (muchas veces opacadas por la rutina diaria); cuestión que puede ser favorecida con el manejo de fuentes documentales que tradicionalmente son consultadas para la elaboración de la historia local, como los registros parroquiales, las actas del ayuntamiento o la prensa local. Todas ellas de cómodo acceso y comprensión (Prats, 1992).

Es evidente que el aprendizaje de las técnicas de los historiadores relacionadas con la búsqueda de información, análisis de los contenidos y obtención de conclusiones a partir de hipótesis iniciales refuerza la formación de los profesionales de la Educación. Se considera necesario convertir el método del historiador en un contenido escolar básico, con el que se aborden temáticas relacionadas con la historia local y/o comarcal permitiendo investigar el entorno de 
cara a construir un conocimiento histórico. La utilización de la historia local puede ser muy edificante para el niño. Se trata de partir de la historia de agentes sociales e históricos con los que se comparten prácticas ordinarias de la vida cotidiana. Partir de contenidos próximos al medio en el que vivimos permite crear espacios de identificación, proximidad y cercanía hacia la historia y sus actores. Cabe señalar por último cómo el resultado de estas investigaciones locales puede dar a los discentes una visión global de la ocupación del territorio, de los recursos, así como de los problemas actuales, ya sean de índole económica, social, política, cultural o religiosa.

Por eso mismo hay que otorgar a los docentes en formación de Educación Primaria herramientas para que su actividad futura en el aula pueda desarrollar este tipo de procedimientos tan valiosos para la Didáctica de las Ciencias Sociales. Es cierto que cada vez hay más trabajos que proponen planteamientos para el uso de las fuentes históricas en espacios educativos (Montanares y Llancavil, 2016; López del Amo, 1999; Tribó, 2005; Valle, 2011; Sáiz, 2014), sin embargo aún se debe trabajar más por encontrar propuestas didácticas concretas que permitan al profesorado ponerlas en práctica. Excepciones destacadas en este aspecto, y con el manejo del Catastro de Ensenada, son las realizadas por R. García-Morís (2016) y R. Moreno (2016).

Se trata de una herramienta muy eficaz contra el estudio a través de la memorización, tradicional método de estudio del alumnado y que tan alejado se encuentra del deseado aprendizaje significativo, la cual favorece la construcción del conocimiento desde la integración teórico-práctica. En un espacio de formación de futuros docentes, en educación superior, es necesario implantar dinámicas pedagógicas procedimentales, alejadas de perspectivas memorísticas (Andelique, 2011), que animen al estudiantado a estimular el aprendizaje por medio de la indagación de información, la selección de fuentes, su interpretación y su análisis crítico (Gómez, Ortuño y Molina, 2014; Sáiz y Gómez, 2016; Sáiz y Domínguez, 2017).

$Y$ es que con estas programaciones prevalecen los contenidos que involucran el perfeccionamiento de destrezas de reflexión más complejas. Habilidades, vinculadas al pensamiento histórico, que tienen que estar forzosamente unidas a las capacidades de contar, ya que la narrativa del pasado es un fundamento principal en la enseñanza de la historia (Sant, Pagès, Santisteban, González y Oller, 2014).

Su enseñanza es un elemento de primera magnitud para la formación para una ciudadanía crítica porque, desde el examen de las sociedades pretéritas, se estimula el razonamiento crítico y el análisis del mundo actual. Muy alejado a un aprendizaje donde el docente trata los hechos pasados con poca objetividad con el fin de privilegiar planteamientos políticos e ideológicos actuales (Prats, 1997). Esto último, lamentablemente, resulta más habitual de lo deseado.

Las metodologías utilizadas en la enseñanza de contenidos de Ciencias Sociales necesariamente tienen que incitar que los discentes descubran el medio en el que viven, reconociéndose con su entorno, a la vez que gradualmente va comprendiendo la complejidad propia de la construcción de los procesos históricos (Gómez, Miralles, López y Prats, 2017).

Si se sitúa al alumnado en el centro del proceso de enseñanza-aprendizaje, como el principal protagonista del ejercicio reflexivo, tendrá los instrumentos apropiados para el desarrollo de competencias vinculadas al pensamiento histórico, social y crítico (Domínguez, 2015). En este sentido, la aproximación al oficio de historiador se muestra como un excelente camino para que se alcance correctamente cómo fueron las sociedades pasadas, que a su vez está sujeto a la adquisición de las capacidades propias para comprender e interpretar el pasado de forma autónoma, para establecer su propia representación de la historia y contextualizar los hitos del pasado apropiadamente (Santisteban, 2010).

Por todo ello, es necesario fomentar la enseñanza en pensamiento histórico como herramienta primordial para regenerar el proceso de enseñanza-aprendizaje en los contenidos de historia. 
Seixas y Morton presentaron seis puntos básicos para conseguir el desarrollo de la conciencia histórica, en toda su complejidad. Entre todos ellos destacamos el trabajo con fuentes primarias, como pruebas (o evidencias) históricas de primer orden (Seixas, 2013; Seixas y Morton, 2013), ya que entendemos que la capacidad de reflexionar históricamente también pueden provenir desde una labor crítica con las fuentes documentales (González, 2006).

En esa línea, Santisteban, González y Pagès (2010), afirman que las fuentes documentales son una alternativa justificada al libro de texto porque ayudan a trabajar contenidos de historia familiar y local, aproximarnos a los protagonistas de la vida cotidiana pasada, cavilar sobre la relatividad del conocimiento o preguntarse sobre la objetividad de la historia, transformándose en un utensilio eficaz, asequible y atractivo para el alumnado.

Por este motivo, y porque introduce al método científico, es necesaria la formación del estudiantado del Grado de Educación Primaria a partir de actividades que se acerquen a la realidad y con manejo de documentación histórica. De este modo, se podrá tener conciencia de primera mano de qué forma se construye el conocimiento histórico (Hernández, 2002), se privilegiará el aprendizaje significativo y la formación de una ciudadanía crítica y comprometida. En definitiva, la utilización de las fuentes documentales como recurso didáctico es una buena vía para trabajar todas estas cuestiones (Chaparro, Guerrero y Felices, 2020).

\section{Diseño de la innovación docente}

Desde estos presupuestos, en el curso 2018/2019, se implantó una innovación con el alumnado de tercer curso del grado en Educación Primaria de la Universidad de Córdoba para dar a conocer y estudiar la historia local. Para ello, y para conseguirlo de la manera más interactiva, activa y lúdica posible, se decidió trabajar con el Catastro de Ensenada, más concretamente con las averiguaciones realizadas sobre la ciudad de Córdoba.

Este Catastro es fruto de un ambicioso proyecto fiscal, que fue diseñado por el marqués de la Ensenada, secretario de Estado y del Despacho de Hacienda, para la implantación de la Única Contribución en las provincias de Castilla. Esta medida hacendística generó una cuantiosa documentación, que está siendo conservada en archivos públicos, como el Archivo General de Simancas y los archivos históricos provinciales (Fernández, 1989).

La recogida de datos en cada uno de los pueblos de Castilla se sistematizó en dos niveles, lo que también hace favorable su consulta. El primero corresponde a las Respuestas Generales. Un cuestionario de cuarenta preguntas que proporciona una información muy completa sobre diversos asuntos de la localidad: población, jurisdicciones o producción económica. La otra sección, coloquialmente denominada respuestas particulares, es un amplio y diverso conjunto de documentos sistematizados por los escribanos del Catastro en varios bloques, entre los que se encuentran los memoriales o declaraciones de los cabezas de familia, por ejemplo (Camarero, 2002; Díaz López, 1995).

Desde un punto de vista metodológico, se consultaron las respuestas generales en el repositorio del Portal de los Archivos Estatales Españoles (PARES), con el objeto de enriquecer el proceso mediante recursos motivadores. La plataforma, además de ofrecer numerosa información histórica del Catastro, nos permite técnicamente muchas facilidades para leer la documentación: ampliar, reducir y descargar cada una de las imágenes.

El trabajo con las respuestas generales del Catastro de Ensenada en la formación histórica de los futuros docentes en Primaria tiene multitud de ventajas. 


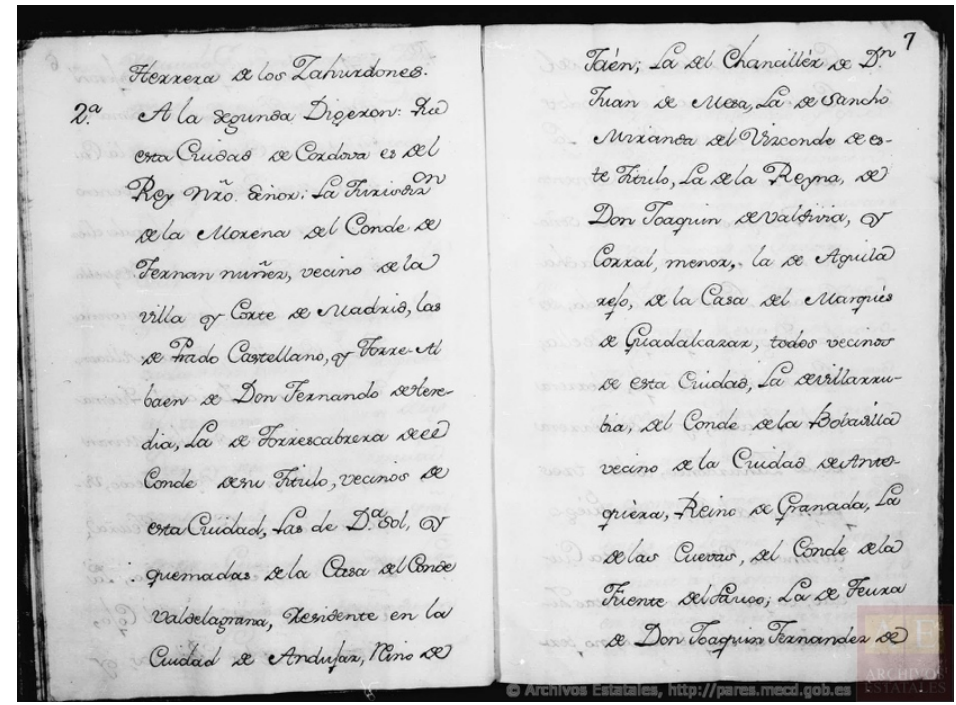

Comienzo de la segunda pregunta del Catastro de Ensenada. Ciudad de Córdoba.

Archivo General de Simancas, Catastro de Ensenada, Respuestas Generales, L.123, fol.007. (PARES)

Por una parte, se permite su acceso público y en formato digital. La caligrafía con la que se escribieron es totalmente legible y la información se presenta con buena letra, grande y espaciada, con los renglones bien separados entre sí, lo que favorece su comprensión. Si hubiera problemas en este sentido, hay que destacar que la editorial Tabapress, en colaboración con la Dirección General del Catastro, editó la Colección Alcabala del Viento, donde están las transcripciones de las respuestas generales de numerosas localidades, que fueron publicadas en varios volúmenes.

Por otro lado, las expresiones utilizadas por los autores para la redacción del Catastro pueden resultar algo extrañas para parte del alumnado, ya que en ocasiones están construidas con vocablos y terminologías totalmente diferente a la actual (como las medidas de longitud o de peso, por ejemplo). Sin embargo, el docente no debe tomar estas cuestiones como un problema, sino como una oportunidad para poder desarrollar una formación mucho más completa de los procesos históricos.

Para guiar adecuadamente las actividades con la documentación, ya que pueden ser algo complicadas por la falta de experiencia del alumnado en tareas investigadoras, se decidió seleccionar las preguntas más adecuadas de las respuestas generales, para la obtención de los objetivos establecidos con anterioridad, que en este caso han estado vinculados principalmente a la construcción de la historia local. También para atender al estudiantado que se le hacía más dificultoso la lectura del documento, se dio como última instancia la posibilidad de consultar la obra de Antonio López Ontiveros (1990), sobre las respuestas generales de la ciudad de Córdoba.

\subsection{Cronograma de la actividad}

Dentro de las actividades concretas, se establecieron seis sesiones, de hora y media cada una, para llevar a cabo el trabajo: la redacción de un texto (sin límite de extensión) con los datos importantes sobre la ciudad de Córdoba en el siglo XVIII, respondiendo a preguntas seleccionadas del Catastro de la Ensenada. Todas ellas fueron concluidas con una puesta en común con la intención de fomentar el trabajo colaborativo. En esos momentos se pudieron compartir dudas, sugerencias e información, con el interés de que fuera útil a la hora de la redacción final del trabajo: 
Sesión 1

Se establecieron los grupos de trabajo (de 4 o 5 personas), además de realizarse una presentación de la práctica, explicando la documentación, estableciendo las primeras pautas de investigación y organizando el trabajo de los días venideros. De manera concreta, a lo largo de la primera sesión el alumnado hubo de aproximarse a la fuente, tratando de comprender las características de la documentación (estructura, tipo de letra, terminología,...), así como del manejo del portal PARES.

\section{Sesión 2}

El alumnado debió realizar la lectura, transcripción y análisis de las primeras preguntas del Catastro de la Ensenada escogidas por el docente:

-1. Cómo se llama la población.

-2. Si es de realengo o de señorío, a quién pertenece, qué derechos percibe y cuánto produce.

-3. Qué territorio ocupa el término, cuánto de levante a poniente y del norte al sur, y cuánto de circunferencia, por horas, y leguas, qué linderos o confrontaciones; y qué figura tiene, poniéndola al margen.

-11 . Qué especies de frutos se cogen en el término.

-17 . Si hay algunas minas, salina, molinos harineros u de papel, batanes u otros artefactos en el término, distinguiendo de qué metales y de qué uso, explicando sus dueños y lo que se regula produce cada uno de utilidad al año.

Sesión 3

El estudiantado continuó con la lectura, transcripción y análisis de las segundas preguntas seleccionadas por el docente:

- 18. Si hay algún esquilmo en el término, a quien pertenece, qué número de ganado viene al esquileo a él y que utilidad se regula da a su dueño cada año.

- 19. Si hay colmenas en el término, cuántas y a quien pertenecen.

- 21. De qué número de vecinos se compone la población y cuántos en las casas de campo o alquerías.

Sesión 4

Se continuó desarrollando la misma labor con estos apartados:

-22. Cuántas casas habrá en el pueblo, qué número de inhabitables, cuántas arruinadas; y si es de señorío, explicar si tienen cada una alguna carga que pague al dueño por el establecimiento del suelo, y cuánto.

-28. Si hay algún empleo, alcabala u otras rentas enajenadas, a quién, si fue por servicio pecuniario u otro motivo, de cuánto fue y lo que produce cada uno al año, de que se deberán pedir los títulos y quedarse con copia.

-29. Cuántas tabernas, mesones, tiendas, panaderías, carnicerías, puentes, barcas sobre ríos, mercados, ferias, etc. hay en la población.

- 30. Si hay hospitales, de qué calidad, qué renta tienen y de qué se mantienen.

\section{Sesión 5}

Tras el análisis y transcripción de dichas preguntas, se llevó a cabo el trabajo de la ubicación de los lugares y monumentos más significativos que aparecen en las fuentes histórica y pudieron 
apoyarse en bibliografía sobre la historia local de la Ciudad, entre otros recursos.

\section{Sesión 6}

Se terminó la actividad con la elaboración de un itinerario por el casco histórico, con el objetivo de conocer la realidad actual de los espacios que aparecen en el Catastro y llevar al alumnado a reflexionar sobre lo aprendido.

\section{Metodología}

\subsection{Objetivos}

Una vez que estuvieron bien concretados y constituidos los períodos de la actividad con el Catastro de Ensenada, se formularon una serie de objetivos generales, específicos y didácticos:

Objetivos Generales:

- Conocer la percepción de los futuros docentes sobre la innovación educativa que favoreciera la mejora de la calidad de la enseñanza de contenidos de Ciencias Sociales, por medio del trabajo con fuentes documentales.

- Plantear estrategias metodológicas que pongan en valor la enseñanza de la historia local en el aula de Educación Primaria.

Objetivos específicos:

- Estimar el comportamiento de los alumnos y alumnas utilizando un recurso como las fuentes históricas como elemento transmisor de contenidos.

- Confirmar si la metodología pedagógica aplicada ha sido eficaz a la hora del aprendizaje de la historia local.

- Evaluar la mejora o no del desarrollo de la conciencia histórica a través del trabajo con las fuentes históricas primarias.

- Valorar el interés del uso de las fuentes históricas primarias, así como otros recursos disponibles en archivos en su labor como futuros maestros y maestras.

- Analizar los grados de motivación e interés que provocan el trabajo con fuentes documentales en el alumnado de Educación Primaria.

Objetivos didácticos:

- Adentrar al estudiantado en el estudio de la historia local como recurso para la enseñanza.

- Despertar la curiosidad en el alumnado por la exploración histórica del entorno, fomentando el interés por el descubrimiento del pasado.

- Subrayar al alumnado que los documentos relacionados con su ciudad son fuentes interesantes para ser utilizadas para la comprensión de la historia.

- Destacar el protagonismo de las fuentes documentales históricas en la metodología de la historia.

- Instruir a los discentes con la metodología propia del conocimiento histórico.

- Habituar al estudiantado en el acceso y manejo de plataformas digitales con fondos documentales en Internet.

- Desarrollar destrezas vinculadas con el tratamiento de la información obtenida en los documentos históricos y con las TIC. 


\subsection{Participantes}

La investigación se cometió sobre una muestra potencial de 50 matriculados en la asignatura Didáctica de las Ciencias Sociales. Se trató de un total de 24 personas $(n=24)$, es decir, un $48 \%$ del alumnado matriculado, donde el $56,25 \%$ eran mujeres y el $43,75 \%$, hombres. Asimismo, el $70 \%$ del total tiene su lugar de residencia en la ciudad de Córdoba.

\subsection{Instrumento}

Con el fin de recabar datos que, tras ser analizados, nos posibilitaran la construcción de conclusiones rigurosas sobre las percepciones del alumnado en torno a la innovación efectuada, se diseñó un cuestionario compuesto por con un total de 20 preguntas (a través de un formulario de Google Form), no validado por expertos, con preguntas de carácter abierto y cerrado. El análisis de los resultados obtenidos posibilitó realizar valoraciones tanto de corte cuantitativo como cualitativo acerca de las competencias desarrolladas a través de la actividad y su percepción sobre lo apropiado del uso de las fuentes documentales para el aprendizaje de la historia local.

Para el examen y análisis de la información recabada se utilizó el paquete estadístico SPSS, versión 25.0 para Windows, que proporcionó la obtención de los siguientes resultados.

\section{Resultados}

En general, la inmensa mayoría del alumnado encuestado reaccionó positivamente ante la actividad propuesta con las respuestas generales del Catastro de Ensenada. De este modo, un $95,8 \%$ respondió que le había parecido interesante y didáctica la tarea propuesta con las fuentes documentales (Catastro de Ensenada), mientras que sólo un estudiante $(4,2 \%)$ apreció lo contrario.

\section{3. ¿Te ha parecido interesante y educativa la actividad? \\ 24 respuestas}

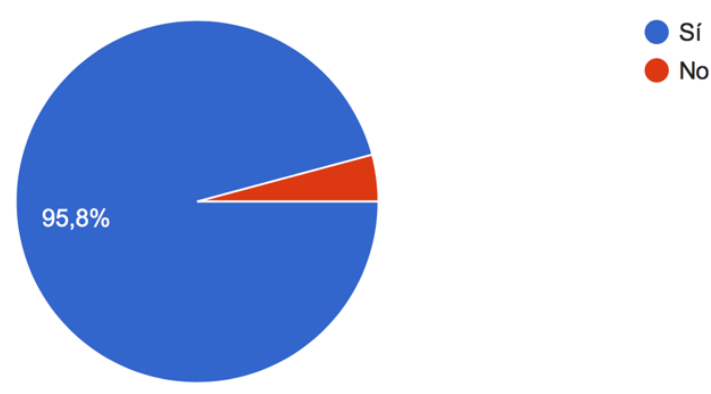

Niveles de interés del alumnado por la actividad. Elaboración propia

Estas contestaciones fueron resultado de haber planteado la actividad sobre la historia local de Córdoba. A todos ellos les pareció interesante y enriquecedora porque "te permite aprender nuevos conocimientos sobre la Córdoba del pasado y al trabajar con nuestra propia ciudad nos genera motivación para continuar aprendiendo" (alumno 5); "comprendemos mejor la historia de nuestra ciudad" (alumno 7); "porque me ha ayudado a conocer más aspectos históricos de mi ciudad" (alumno 11); "porque he conocido sitios y monumentos que antes no tenía idea de su existencia, o muy poca. Y es una manera más entretenida de aprender sobre Córdoba" (alumno 8); "porque no conocemos nada de nuestra ciudad y es importante que aprendamos 
cosas sobre ella para utilizarlo como futuros maestros" (alumno 14); "porque me ha permitido conocer lugares de mi ciudad que antes no conocía, además, ha supuesto un acercamiento entre la época antigua y la actual" (alumno 16); o "he descubierto lugares que a pesar de haber pasado por ellos no sabían lo que representaban" (alumno 20).

De hecho, una gran mayoría de estudiantado (75\%) estimó que sus conocimientos sobre la historia de Córdoba tras haber realizado la tarea habían aumentado a un nivel "medio-alto", a la vez que un $25 \%$ piensa que es "medio-bajo".

1. ¿Cómo calificarias tu conocimiento de la Córdoba del siglo XVIII después de haber realizado la actividad? (monumentos, personajes, edificios...)

24 respuestas

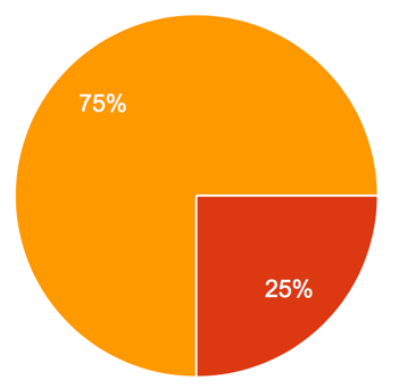

Bajo

Medio-Bajo

Medio-Alto

Alto

Niveles de conocimiento de la historia de Córdoba del alumnado tras la realización de la actividad. Elaboración propia

Por otro lado, el $62,5 \%$ de los discentes entendió que sus competencias de análisis de fuentes históricas habían mejorado notablemente después de haber cumplido con la práctica, mientras que un $37,5 \%$ pensó que su capacidad todavía no era "alta".

16. Esta tarea ha estado fundamentada en fuentes históricas, ¿Cuál es tu nivel de competencia para trabajar con ellas después de haber realizado la actividad?

24 respuestas
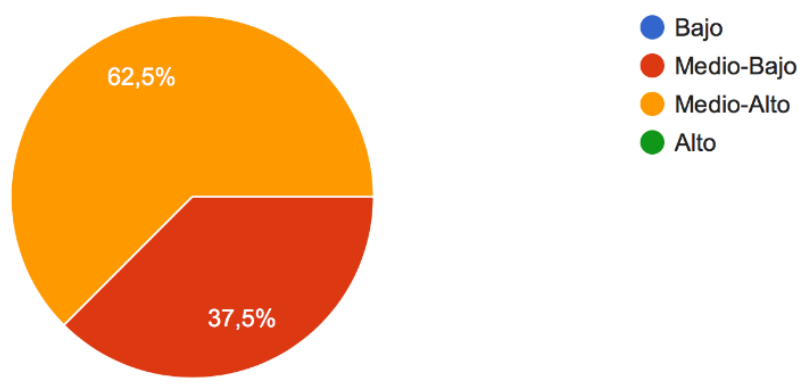

Niveles de competencia del alumnado tras la realización de la actividad. Elaboración propia

A este respecto, en lo referente al trabajo con fuentes documentales, el estudiantado por completo afirmó que en el futuro, durante su desarrollo profesional, iba a valorar trabajar con ellos como recurso didáctico. Todo ello porque, por ejemplo, los documentos permiten formar 
críticamente (alumno 4), además de ser una actividad muy dinámica (alumno 10) y porque de ellos se puede extraer mucha más información de la que ofrece Internet (alumno 14).

Por este motivo, el 95,8\% concibió que había desarrollado su conciencia histórica, aumentando su capacidad de pensar la historia local gracias a la práctica con las respuestas generales del Catastro de Ensenada. Respecto a la formación de una ciudadanía crítica, algún estudiante estimó que la tarea ayudaba a promover los valores cívicos y la solidaridad, como son los relacionados con la conservación del patrimonio histórico (alumno 19).

5. Uno de los principales objetivos de la actividad ha sido desarrollar nuestra conciencia histórica ¿Crees que ha mejorado tu capacidad de pensar la historia de tu ciudad a través de esta práctica? 24 respuestas

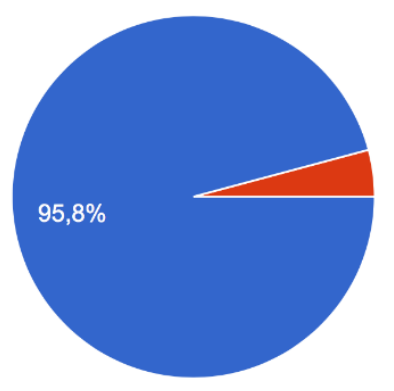

Sí

No

Niveles de desarrollo de la conciencia histórica del alumnado tras la realización de la actividad. Elaboración propia

Cuando se le instó a enumerar algunos de los archivos que se encuentran en la ciudad de Córdoba, el $16,7 \%$ no supo dar contestación, mientras que el $45,8 \%$ respondió con algún dato apropiado: principalmente el Archivo Municipal de Córdoba (alumnos 2, 4, 9, 10, 11, 12, 13, 14, 16, 17 y 18). Los tres últimos también añadieron el Archivo de la Catedral de Córdoba. El resto de los encuestados $(37,5 \%)$ ofrecieron datos equivocados.

Como la actividad estuvo íntimamente relacionada con la utilización con repositorios documentales de Internet, los estudiantes tuvieron la oportunidad de expresarse sobre esta cuestión. En este sentido, un 95,8\%, casi la totalidad, se manifestó a favor de estas plataformas digitales que ofrecen la consulta de fuentes históricas y que las tendrán en cuenta en su quehacer como maestros. Son diversos los motivos, pero principalmente destacaron que "es un buen recurso didáctico" (alumno 11); "fácil acceso y asequible desde cualquier lugar" (alumno 1); "a disposición de todos y porque además, al estar digitalizados, es más fácil conseguirlos y trabajar con ellos" (alumno 16); es "muy útil" (alumnos 6, 7, 9, 13); favorece el trabajo de los "documentos de nuestra historia" (alumno 14, 18, 19); o "es necesario las nuevas tecnologías para complementar la explicación" (alumno 4). Al contrario, sólo a un estudiante no le pareció adecuado el manejo como de este tipo de webs: "resulta un poco complicado" (alumno 10).

\section{Discusión}

En un primer momento, cabe destacar que la generalidad de los encuestados se expresó favorable a la innovación. Este asunto nos lleva a reflexionar sobre la necesidad de implantar técnicas innovadoras de enseñanza en las aulas universitarias, que también conlleven la 
introducción de metodologías activas de aprendizaje de contenidos sociales durante las etapas educativas obligatorias.

Respecto a los resultados de la actividad realizada, cabe destacar que la mayoría de los participantes (95\%) expresó que ha mejorado su capacidad de pensar históricamente tras la realización de la actividad. Se trata de una respuesta muy positiva, pero es cierto que tenemos que recapacitar sobre cuál es el motivo de que lo comprendan así. Según la información recopilada, nos hace pensar que lo entienden de este modo porque se han dado cuenta que saben más de la historia de la ciudad de Córdoba que antes. Y así lo atestigua la investigación que, en general, muestra por parte de los discentes un amplio desconocimiento sobre este tema.

Con todo, y sin ninguna duda, este es el camino a seguir porque si se le otorga las herramientas necesarias (en especial, fuentes documentales), y ayudado por el docente para la consulta y el ejercicio de análisis, el alumnado será capaz de pensar y construir la Historia en su complejidad y críticamente.

En este sentido, en lo que se refiere a "lo concreto", resultó de gran importancia haber podido trabajar con el entorno próximo, lo que demuestra el valor de la historia local como una herramienta de primer nivel para la Didáctica de las Ciencias Sociales.

Así lo expresaron: "Me ha gustado mucho el trabajo y me ha parecido muy interesante ya que hemos aprendido mucho de Córdoba" (alumno 5); "me ha parecido novedosa, muy útil para nuestra vida porque nos permite conocer la historia de nuestra ciudad" "y en un futuro puede ser usadas cuando seamos docentes" (alumno 17); o "me ha parecido interesante y algo nuevo que hasta ahora no había probado para conocer la historia de Córdoba" (alumno 4).

Respecto al uso de fuentes históricas como recurso didáctico para el aprendizaje de la Historia, la mayoría del alumnado $(62,5 \%)$ opinó que sus competencias para su lectura y análisis había aumentado notablemente. Es un dato muy interesante porque nos demuestra que personas no vinculadas estrechamente con la historia son competentes para leer y analizar las fuentes documentales propias del pasado.

Dicho de otro modo: se demuestra que cada uno de nosotros podríamos ser capaces de investigar, estudiar y construir a través de la consulta de fuentes primarias, tanto los orígenes y causas de los hechos históricos como las configuraciones y evoluciones de las sociedades del pasado (procesos, dinámicas, instituciones, ...). Por lo tanto, no hay impedimento para usar los documentos como recurso para la construcción del pensamiento histórico y crítico; procedimientos que van mucho mejor si se hacen acompañados con reflexiones sobre la sociedad actual.

La utilización de las nuevas tecnologías en el aula siempre suele ser recibida por el estudiantado de forma positiva y así ha sido ocurrido con los repositorios documentales de Internet, declarando que los usarán cuando ejerzan de maestros y maestras en el aula. Sin embargo, entendemos que tras estas afirmaciones tan positivas se esconde otra realidad, la cual también se contempla en la investigación realizada: por parte del estudiantado existe un gran desconocimiento sobre archivos (tanto de los estatales como de los provinciales o municipales) y esto comporta que tampoco saben qué tipo de documentación se puede albergar allí y qué labor desempeñan los profesionales que allí trabajan. Si no se conocen sus características es más difícil implantar metodologías activas y renovadoras en el aula.

Por este motivo, es muy importante establecer vínculos duraderos entre los profesionales de la Educación con los historiadores y técnicos de archivos para establecer asociaciones positivas para todos, que conformen acciones para la realización propuestas específicas, bien diseñadas, realistas, contextualizadas en el curriculum y accesibles al alumnado. 


\section{Conclusiones}

La actividad renovadora desarrollada con el alumnado del Grado de Primaria ha resultado del todo positiva, gracias una planificación centrada en dos instrumentos muy atractivos: trabajar con la historia local (el entorno, con aquello que les es cercano y se convierte en algo más atractivo) y el aprovechamiento de herramientas diferentes a las tradicionales, como fue el trabajo con fuentes documentales, que se vieron reforzadas con el uso de las TIC.

La experiencia desarrollada con el trabajo con el Catastro de Ensenada favoreció la motivación por el aprendizaje del estudiantado, ya que la consulta de documentos históricos desde dispositivos electrónicos despertó su curiosidad. Sin embargo, no debemos olvidar que lo que más le inquieta es participar en actividades que tengan que ver con su entorno: adquirir conocimientos sobre su ciudad, realizar itinerarios didácticos por ellos, etc., por lo que supone por enriquecimiento personal y porque entienden que es útil para su futura labor como docentes en Educación Primaria. Así se puede comprobar cuando repasamos sus respuestas, lo que nos indica que como docentes de maestros en formación es primordial ser imaginativos, a la vez que rigurosos, para impulsar a nuestro alumnado en el pensamiento crítico.

Estas prácticas servirán como base para que puedan reflexionar sobre la importancia de enseñar para la formación de una ciudadanía crítica. Con todo, y para conseguir resultados óptimos, es importante que el docente ajuste los contenidos de manera conveniente y que consiga que los documentos sean inteligibles y de fácil acceso para los estudiantes.

Por todo ello, el conocimiento del entorno y la historia local son unas herramientas extraordinarias para la interpretación del pasado más cercano. Igualmente, son muy válidas para la construcción gradual de la propia identidad y surten de destrezas para la participación en el medio social y cultural.

\section{Referencias bibliográficas}

Aktekin, S. (2010). The place and importance of Local History in the Secondary History Education. Eğitimde Kuram ve Uygulama-Journal of Theory and Practice in Education, 6 (1), 86-105.

Andelique, C. M. (2011). La didáctica de la historia y la formación docente: ¿qué profesor de historia necesitan las escuelas? Clío \& Asociados, 15, 256-269.

Camarero, C. (2002). El Catastro de Ensenada, 1745-1756. Diez años de intenso trabajo y 80.000 volúmenes manuscritos. CT. Catastro, 46, 61-88.

Chaparro, Á.; Guerrero, R. y Felices, M. M. (2020). Humanizar la enseñanza de las Ciencias Sociales a través de fuentes históricas. En Felipe, A. (Coord.). Estudios sobre innovaciones educativas. Madrid: Pirámide.

Díaz López, J. P. (1995). La Única Contribución como objetivo de política ilustrada: la

realización del Catastro de Ensenada en el Valle de Andarax. Boletín del Instituto de Estudios Almerienses,14, 105-127.

Domínguez, J. (2015). Pensamiento histórico y evaluación de competencias. Barcelona: Graó.

Domínguez, J. (1986). La historia local en la escuela. En Domínguez, J. Vidaechea, T. (Coords.) EI entorno en las Ciencias Sociales. Segovia: Secretaría General Técnica. Centro de Publicaciones. Ministerio de Educación y Ciencia, 63-74.

Fernández, F. (1989). Las respuestas particulares al Catastro de Ensenada y su explotación mediante microordenador y el lenguaje dBASE. Norba. Revista de geografía, 8-9, 539550 . 
García-Morís, R. (2016). Propuesta metodológica para el uso de las fuentes históricas demográficas de la Edad Moderna como recurso didáctico. Revista de Didácticas Específicas, 14, 71-85.

Gómez, C. J.; Miralles, P.; López, R. y Prats, J. (2017). Las competencias históricas en el horizonte. Propuestas presentes y perspectivas de futuro. En López, R.; Miralles, P.; Prats, J. (Ed.). Enseñanza de la historia y competencias educativas. Barcelona: Graó, 2015-227.

Gómez, C.J.; Ortuño, J. y Molina, S. (2014). Aprender a pensar históricamente. Retos para la historia en el siglo XXI. Revista Tempo e Argumento, Florianópolis, n¹1 (6), 5-27.

Gómez, C.J. y García Gónzález, F. (2018). Identidad, patrimonio y pensamiento histórico. ARBOR. Ciencia, Pensamiento y Cultura, vol. 194-788, abril-junio, 1-4.

López del Amo, M. I. (1999). La utilización didáctica de las fuentes históricas en el Bachillerato. Iber: Didáctica de las ciencias sociales, geografía e historia, 20, 81-90.

López Ontiveros, A. (1990). Córdoba 1752 según las respuestas generales del Catastro de Ensenada, Ed. Tabapress.

Montanares-Vargas, E. y Llancavil-Llancavil, D. R. (2016). Uso de fuentes históricas en formación inicial de profesores. Magis, Revista Internacional de Investigación en Educación, 8 (17), 85-98.

Moral, C., Pérez, P., Torremocha, M.A. Valls, R. (1999). La enseñanza de la Historia: España. Bogotá.

Moreno, R. (2016). El Catastro de Ensenada, al alcance del aula. Iber: Didáctica de las ciencias sociales, geografía e historia, 85, 64-68.

Pagès, J. (2000). La formación inicial del profesorado para la enseñanza del patrimonio histórico y de la historia. Treballs d'Arqueologia, 6, 205-217.

Pedreño Ros, D. (2015). Aportaciones Didácticas de la Historia Local y Familiar al Proceso de Enseñanza-Aprendizaje de las Ciencias Sociales. Experiencias en Tres Centros Educativos de la Región de Murcia de Educación Primaria, Secundaria y PCPI. Tesis defendida en la Universidad de Murcia. URI: http://hdl.handle.net/10201/46981.

Piaget, J. (1977). The role of action in the development of thinking. Knowledge and development, vol. 1, 17-42.

Prats, J. (1996). El estudio de la Historia local como opción didáctica. ¿destruir o explicar la historia. Iber Didáctica de las ciencias sociales, geografía. e historia, 8, 93-106.

Rogers, A. (1972). Approaches to Local History. London-New York: Ed. Longman.

Sáiz, J. (2014). Fuentes históricas y libros de texto en secundaria: una oportunidad perdida para enseñar competencias de pensamiento histórico. Ensayos, Revista de la Facultad de Educación de Albacete, 29 (1), 83-99.

Sáiz, J. y Domínguez, J. (2017). Aprender sobre la historia: competencias metodológicas en educación secundaria. En López, R.; Miralles, P., Prats, J. y Gómez, C. J. (Coords.). Enseñanza de la historia y competencias educativas. Barcelona: Editorial Graó, 23-48.

Sáiz, J. y Gómez, C. J. (2016). Investigar el pensamiento histórico y narrativo en la formación del profesorado: fundamentos teóricos y metodológicos. Revista electrónica interuniversitaria de formación de profesorado, 19 (1), 175-190. 
Sant, E.; Pagès, J.; Santisteban, A., González-Monfort, N. y Oller, M. (2014). ¿Cómo podemos analizar la competencia narrativa del alumnado en el aprendizaje de la historia?, Clío \& Asociados, 18-19, 166-182.

Santisteban, A. (2010). La formación de competencias de pensamiento histórico. Clio \& asociados: La historia enseñada, 14, 34-56.

Santisteban, A. González, N. y Pagès, J. (2010). Una investigación sobre la formación del pensamiento histórico. Ávila, R. Mạ., Rivero, Mạ. P. y Domínguez, P. L. (Coords.). Metodología de investigación en Didáctica de las Ciencias Sociales. Zaragoza: AUPDCS, 115-128.

Seixas, P. (2013). Linking Historical Thinking Concepts, Content and Competencies. National Meeting of The Historical Thinking Project. Toronto: University of British Columbia.

Seixas, P. y Morton, T. (2013). The Big Six Historical Thinking Concepts. Toronto: Nelson Education.

Tejada, L. (2009). Las salidas, un recurso para el aprendizaje en educación infantil. Innovación y experiencias educativas. Revista digital, 14, 1-11.

Tribó, G. (2005). Enseñar a pensar históricamente. Los archivos y las fuentes documentales en la enseñanza de la Historia. Barcelona: Horsori.

Valle, A. (2011). El uso de las fuentes escritas en la enseñanza de la Historia. Análisis de textos escolares para tercero y cuarto de secundaria. Educación, 20(38), 81-106.

Vázquez de Prada Vallejo, V. (1987) La historia local: su interés actual. En Tokiko historiaz ikerketak. Estudios de historia local. Donostia-San Sebastián: Eusko lkaskuntza, 9-15.

Vega, L. (1997). La reforma educativa en España (1970-1990). Educar em Revista, 13. 